\title{
碘催化苯乙醛与苄胺合成多取代咪唑的反应机理研究
}

\author{
张 林 ${ }^{a}$ 郑 妍 ${ }^{a}$ 潘晓晓 ${ }^{a}$ 李来才*,a 田安民 ${ }^{b}$ \\ $\left({ }^{a}\right.$ 四川师范大学化学与材料科学学院 成都 610066) \\ $\left({ }^{b}\right.$ 四川大学化学学院 成都 610064)
}

\begin{abstract}
摘要 采用密度泛函理论(DFT)中的 B3LYP 方法, 在 6-31+G(d)基组水平下, 研究了碘催化苯乙醛和芐胺发生氧化环 化合成多取代咪唑的微观反应机理.对反应通道上反应物、中间体、过渡态和产物进行了结构优化，对优化后的各化合 物在 B3LYP/6-311+ $+\mathrm{G}(\mathrm{d}, \mathrm{p})$ 基组下进行单点能计算和零点能桥正, 通过振动频率分析及内毫反应坐标(IRC)计算对过 渡态进行了验证, 并运用自然键轨道理论(NBO)和分子中的原子理论(AIM)分析了化合物的轨道间相互作用及成键特 征. 研究结果发现: 无碘催化时, 苯乙醛和芐胺发生氧化环化合成多取代咪唑,其反应速控步骤活化能为 $514.32 \mathrm{~kJ} \cdot$ $\mathrm{mol}^{-1}$, 在硒催化作用下, 反应速控步骤活化能为 $145.94 \mathrm{~kJ}^{\circ} \mathrm{mol}^{-1}$. 比较研究结果, 说明碘能有效催化该反应的进行, 碘的 催化性能主要表现在活化苯乙醛乙基上的 C- $\mathrm{H}$ 键. 我们还采用连续介质模型( $\mathrm{PCM}$ )比较研究了 4 种溶剂化作用对反应 的影响, 得到的研究结果与实验相吻合. 理论预测了有机溶剂二甲亚砜(DMSO)能有效地提高反应产率.
\end{abstract}

关键词＼cjkstart碘; 多取代咪唑; 反应机理; 溶剂化; 密度泛函理论

\section{Investigation the Reaction Mechanism from Phenylacetaldehyde and Benzylamine to Polysubstituted Imidazole Catalyzed by $\mathrm{I}_{2}$}

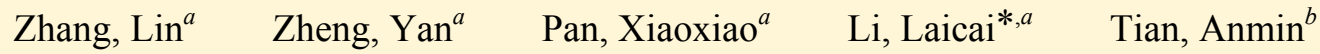 \\ $\left({ }^{a}\right.$ College of Chemistry and Material Science, Sichuan Normal University, Chengdu 610066) \\ ( ${ }^{b}$ College of Chemistry, Sichuan University, Chengdu 610064)
}

\begin{abstract}
The reaction mechanism from phenylacetaldehyde and benzylamine to polysubstituted imidazole catalyzed by $\mathrm{I}_{2}$ was studied by the density functional theory. All of the reactants, intermediates, transition states and product were optimized at B3LYP/6-31 +G(d) level. The single point energy and zero point energy correction were calculated for the optimized configuration of each compound at B3LYP/6-311+ $+\mathrm{G}(\mathrm{d}, \mathrm{p})$ level. Transition states have been confirmed via vibration analysis and intrinsic reactions coordinate (IRC), and nature bond orbital (NBO) and atoms in molecules (AIM) theories have been used to analysis orbits interaction and bond natures. Our results showed that the activation energy of the rate-determining step was $514.32 \mathrm{~kJ} \cdot \mathrm{mol}^{-1}$ without $\mathrm{I}_{2}$-catalyzed, however, the activation energy of the rate-determining step was $145.94 \mathrm{~kJ} \bullet \mathrm{mol}^{-1}$ with $\mathrm{I}_{2}$-catalyzed. It indicated that $\mathrm{I}_{2}$ catalyst promoted reaction effectively, and the $\mathrm{C}-\mathrm{H}$ bond of ethyl of phenylacetaldehyde was activated by $\mathrm{I}_{2}$. In addition, polarized continuum model (PCM) method was adopted to discuss the effects of solvation. All calculations were consistent with experiments. It is predicted that the organic solvent dimethyl sulfoxide (DMSO) can effectively improve the reaction yield.
\end{abstract}

Keywords iodine; polysubstituted imidazoles; reaction mechanism; solvation; density functional theory

咪唑是含有两个间位氮原子的五元杂环化合物，是 一种非中心对称的缺电子环, 广泛存在于生物分子中. 咪唑类化合物具有许多独特的性能，在医学和生物 学 ${ }^{[1,2]}$ 等方面存在巨大的应用潜力, 咪唑环不仅是一些 药物分子的骨架，也是有机合成的中间体，咪唑类化合
物还被广泛用作配体和环境友好离子溶剂 ${ }^{[3]}$. 由于咪唑 类化合物应用广泛，其合成方法也被不断改进. Karami 等 ${ }^{[4]}$ 在 2012 年用 $\mathrm{Fe}_{3} \mathrm{O}_{4}$ 作催化剂合成了一系列新型多取 代咪唑化合物. 史真课题组 ${ }^{[5]}$ 用吲哚乙酸铜催化睛与二 胺合成了 2-咪唑啉. Mazaahir 等 ${ }^{[6]}$ 在 2006 年报道了硒催

\footnotetext{
*E-mail: lilcmail@163.com

Received March 11, 2014; revised April 8, 2014; published online May 5, 2014.

Project supported by the Department of Education of Sichuan Province (No. 13ZA0150).

四川省教育厅重点基金(No.13ZA0150)资助项目.
} 
化四组分一锅法合成咪唑, 最近江焕峰课题组 ${ }^{[7]}$ 也报道 了碘催化芳基酮和芐胺氧化环化合成多取代咪唑. 与金 属催化剂相比, 碘催化剂具有廉价易得、高效稳定、操 作方便、反应条件温和、后处理简单等优点, 近年来碘 催化剂在有机合成 ${ }^{[8]}$ 、绿色化学 ${ }^{[9]}$ 、药物合成 ${ }^{[10]}$ 及新型 催化反应 ${ }^{[11,12]}$ 等方面得到了广泛应用. 因此, 碘催化合 成咪唑类化合物 ${ }^{[10]}$ 具有很高的应用价值, 受到很多课 题组的青睐. 汪志勇小组 ${ }^{[13]}$ 在 2012 年报道了碘催化的 串联氧化环化反应合成多取代咪唑, 反应式如 Eq. 1 所 示. 汪志勇等使用苯乙醛和芐胺为原料, 碘作催化剂, 过氧叔丁醇作氧化剂分别在 $\mathrm{CH}_{3} \mathrm{CN}, \mathrm{C}_{2} \mathrm{H}_{5} \mathrm{OH}$ 等溶剂中 合成了多取代咪唑.

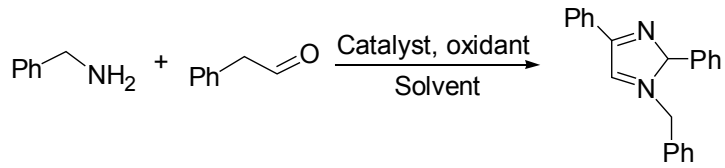

近年来, 理论计算方法被广泛运用于催化剂优化反 应微观机理的研究, 它对反应机理的描述和寻找新反应 通道有着独特的优势, 能很好地解释催化剂的作用机制
和诸多实验现象, 并为实验结果提供可靠的理论数据. Alcaide 等 ${ }^{[14]}$ 采用密度泛函理论研究了 $\mathrm{I}_{2}$ 催化四元氧氮 杂环化合物环扩张的反应机理; Lebœuf 等 ${ }^{[15]}$ 采用密度 泛函方法报道了纳扎罗夫环化反应和瓦格纳-迈尔外因 重排的顺序. 我们课题组 ${ }^{[16]}$ 采用密度泛函理论报道过 过渡金属催化氯代环化反应和 $\mathrm{C}-\mathrm{S}$ 偶联反应 ${ }^{[17]}$ 的微观 反应机理. 理论计算在实验方法的研究中具有重要作 用 ${ }^{[18,19]}$. 因此, 本文采用密度泛函理论深入探讨了碘催 化苯乙醛和茮胺发生氧化环化合成多取代咪唑的微观 反应历程，以揭示碘催化作用机制.

\section{1 结果与讨论}

我们研究了苯乙醛和茮胺氧化环化合成多取代咪 唑反应机理, 分别探讨了有碘催化和无碘催化两种过 程. 反应过程中所有反应物、中间体、过渡态及产物的优 化构型和结构参数分别见图 1 和图 2 , 相关能量见表 1 和表 2. 计算结果表明: 中间体的力常数矩阵本征值均 为正, 是反应势能面上的稳定点, 所有过渡态都具有唯 一的虚频. 过渡态的内禀反应坐标(IRC)计算结果显示 振动趋势分别指向其对应的反应物和产物.

表 1 反应各驻点的相对吉布斯自由能变化 $\Delta G$

Table 1 The Gibbs relative free energy change $\Delta G$ of the compounds in the pathway

\begin{tabular}{|c|c|c|c|}
\hline Species & $\Delta G /\left(\mathrm{kJ}^{\prime} \cdot \mathrm{mol}^{-1}\right)$ & Species & $\Delta G /\left(\mathrm{kJ} \cdot \mathrm{mol}^{-1}\right)$ \\
\hline $\mathrm{R} 1+2 \mathrm{R} 2+2 \mathrm{TBHP}+2 \mathrm{I}_{2}$ & 0.00 & $\mathrm{IM} 16+\mathrm{P} 1+4 \mathrm{HI}+2 \mathrm{H}_{2} \mathrm{O}$ & -136.15 \\
\hline $\mathrm{IM} 1+2 \mathrm{R} 2+2 \mathrm{TBHP}+2 \mathrm{I}_{2}$ & 36.37 & $\mathrm{TS} 10+\mathrm{P} 1+4 \mathrm{HI}+2 \mathrm{H}_{2} \mathrm{O}$ & -121.72 \\
\hline $\mathrm{IM} 2+2 \mathrm{R} 2+2 \mathrm{TBHP}+\mathrm{I}_{2}$ & 32.34 & $\mathrm{P}+\mathrm{P} 1+4 \mathrm{HI}+2 \mathrm{H}_{2} \mathrm{O}+\mathrm{TBHP}$ & -380.04 \\
\hline $\mathrm{TS} 1+2 \mathrm{R} 2+2 \mathrm{TBHP}+\mathrm{I}_{2}$ & 42.25 & $\mathrm{R} 1+2 \mathrm{R} 2+2 \mathrm{TBHP}$ & 0.00 \\
\hline $\mathrm{IM} 3+\mathrm{HI}+2 \mathrm{R} 2+2 \mathrm{TBHP}+\mathrm{I}_{2}$ & 20.42 & $\mathrm{M} 1+2 \mathrm{R} 2+\mathrm{TBHP}$ & 35.88 \\
\hline $\mathrm{IM} 4+\mathrm{HI}+2 \mathrm{R} 2+\mathrm{TBHP}+\mathrm{I}_{2}$ & 18.43 & $\mathrm{~T} 1+2 \mathrm{R} 2+\mathrm{TBHP}$ & 181.31 \\
\hline $\mathrm{TS} 2+\mathrm{HI}+2 \mathrm{R} 2+\mathrm{TBHP}+\mathrm{I}_{2}$ & 141.20 & $\mathrm{M} 2+2 \mathrm{R} 2+\mathrm{H}_{2} \mathrm{O}+\mathrm{TBHP}$ & -208.38 \\
\hline $\mathrm{IM} 5+2 \mathrm{HI}+2 \mathrm{R} 2+\mathrm{TBHP}+\mathrm{I}_{2}$ & 41.88 & $\mathrm{~T} 2+2 \mathrm{R} 2+\mathrm{H}_{2} \mathrm{O}+\mathrm{TBHP}$ & 305.94 \\
\hline $\mathrm{TS} 3+2 \mathrm{HI}+2 \mathrm{R} 2+\mathrm{TBHP}+\mathrm{I}_{2}$ & 187.82 & $\mathrm{IM} 6+\mathrm{P} 2+2 \mathrm{R} 2+\mathrm{H}_{2} \mathrm{O}+\mathrm{TBHP}$ & -281.83 \\
\hline $\mathrm{IM} 6+\mathrm{P} 1+2 \mathrm{HI}+2 \mathrm{R} 2+\mathrm{TBHP}+\mathrm{I}_{2}$ & -211.18 & $\mathrm{IM} 7+\mathrm{P} 2+\mathrm{R} 2+\mathrm{H}_{2} \mathrm{O}+\mathrm{TBHP}$ & -241.95 \\
\hline $\mathrm{IM} 7+\mathrm{P} 1+2 \mathrm{HI}+\mathrm{R} 2+\mathrm{TBHP}+\mathrm{I}_{2}$ & -207.28 & $\mathrm{TS} 4+\mathrm{P} 2+\mathrm{R} 2+\mathrm{H}_{2} \mathrm{O}+\mathrm{TBHP}$ & -132.94 \\
\hline $\mathrm{TS} 4+\mathrm{P} 1+2 \mathrm{HI}+\mathrm{R} 2+\mathrm{TBHP}+\mathrm{I}_{2}$ & -98.27 & $\mathrm{IM} 8+\mathrm{P} 2+\mathrm{R} 2+\mathrm{H}_{2} \mathrm{O}+\mathrm{TBHP}$ & -254.55 \\
\hline $\mathrm{IM} 8+\mathrm{P} 1+2 \mathrm{HI}+\mathrm{R} 2+\mathrm{TBHP}+\mathrm{I}_{2}$ & -219.88 & $\mathrm{TS} 5+\mathrm{P} 2+\mathrm{R} 2+\mathrm{H}_{2} \mathrm{O}+\mathrm{TBHP}$ & -127.05 \\
\hline $\mathrm{TS} 5+\mathrm{P} 1+2 \mathrm{HI}+\mathrm{R} 2+\mathrm{TBHP}+\mathrm{I}_{2}$ & -92.39 & $\mathrm{IM} 9+\mathrm{P} 2+\mathrm{R} 2+2 \mathrm{H}_{2} \mathrm{O}+\mathrm{TBHP}$ & -292.28 \\
\hline $\mathrm{IM} 9+\mathrm{P} 1+2 \mathrm{HI}+\mathrm{R} 2+\mathrm{H}_{2} \mathrm{O}+\mathrm{TBHP}+\mathrm{I}_{2}$ & -234.03 & $\mathrm{IM} 10+\mathrm{P} 2+2 \mathrm{H}_{2} \mathrm{O}+\mathrm{TBHP}$ & -242.51 \\
\hline $\mathrm{IM} 10+\mathrm{P} 1+2 \mathrm{HI}+\mathrm{H}_{2} \mathrm{O}+\mathrm{TBHP}+\mathrm{I}_{2}$ & -207.84 & $\mathrm{TS} 6+\mathrm{P} 2+2 \mathrm{H}_{2} \mathrm{O}+\mathrm{TBHP}$ & -112.20 \\
\hline $\mathrm{TS} 6+\mathrm{P} 1+2 \mathrm{HI}+\mathrm{H}_{2} \mathrm{O}+\mathrm{TBHP}+\mathrm{I}_{2}$ & -77.53 & $\mathrm{IM} 11+\mathrm{P} 2+2 \mathrm{H}_{2} \mathrm{O}+\mathrm{TBHP}$ & -212.93 \\
\hline $\mathrm{IM} 11+\mathrm{P} 1+2 \mathrm{HI}+\mathrm{H}_{2} \mathrm{O}+\mathrm{TBHP}+\mathrm{I}_{2}$ & -178.26 & $\mathrm{TS} 7+\mathrm{P} 2+2 \mathrm{H}_{2} \mathrm{O}+\mathrm{TBHP}$ & -87.43 \\
\hline $\mathrm{TS} 7+\mathrm{P} 1+2 \mathrm{HI}+\mathrm{H}_{2} \mathrm{O}+\mathrm{TBHP}+\mathrm{I}_{2}$ & -52.76 & $\mathrm{IM} 12+\mathrm{P} 2+3 \mathrm{H}_{2} \mathrm{O}+\mathrm{TBHP}$ & -109.54 \\
\hline $\mathrm{IM} 12+\mathrm{P} 1+2 \mathrm{HI}+2 \mathrm{H}_{2} \mathrm{O}+\mathrm{TBHP}+\mathrm{I}_{2}$ & -144.42 & $\mathrm{M} 3+\mathrm{P} 2+3 \mathrm{H}_{2} \mathrm{O}$ & -226.69 \\
\hline $\mathrm{IM} 13+\mathrm{R} 1+2 \mathrm{R} 2+\mathrm{TBHP}+\mathrm{I}_{2}$ & 12.06 & $\mathrm{~T} 3+\mathrm{P} 2+3 \mathrm{H}_{2} \mathrm{O}$ & -62.28 \\
\hline $\mathrm{TS} 8+\mathrm{R} 1+2 \mathrm{R} 2+\mathrm{TBHP}+\mathrm{I}_{2}$ & 150.23 & $\mathrm{M} 4+\mathrm{P} 2+4 \mathrm{H}_{2} \mathrm{O}$ & -449.00 \\
\hline $\mathrm{IM} 14+\mathrm{R} 1+2 \mathrm{R} 2+\mathrm{TBHP}+\mathrm{I}_{2}+\mathrm{HI}$ & 106.65 & $\mathrm{~T} 4+\mathrm{P} 2+4 \mathrm{H}_{2} \mathrm{O}$ & -429.77 \\
\hline $\mathrm{IM} 15+\mathrm{P} 1+3 \mathrm{HI}+2 \mathrm{H}_{2} \mathrm{O}$ & -131.82 & $\mathrm{P} 1+\mathrm{P}+\mathrm{P} 2+4 \mathrm{H}_{2} \mathrm{O}$ & -688.27 \\
\hline $\mathrm{TS} 9+\mathrm{P} 1+3 \mathrm{HI}+2 \mathrm{H}_{2} \mathrm{O}$ & -27.85 & & \\
\hline
\end{tabular}




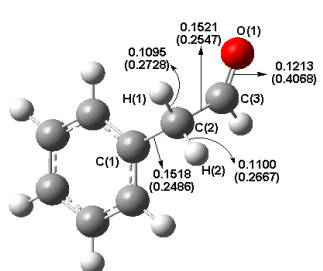

R1

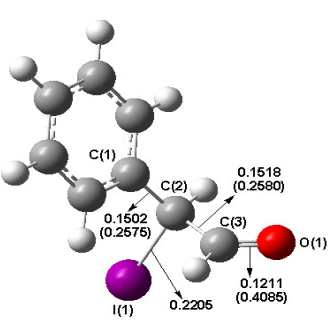

IM3

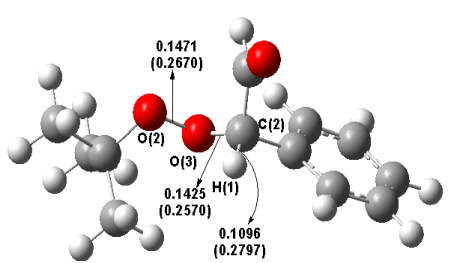

IM5

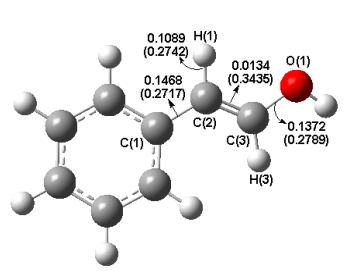

IM1

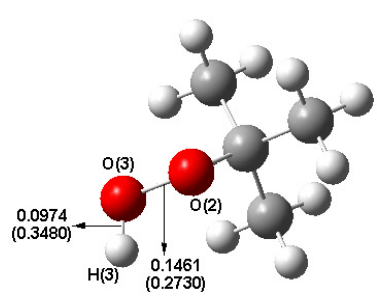

TBHP

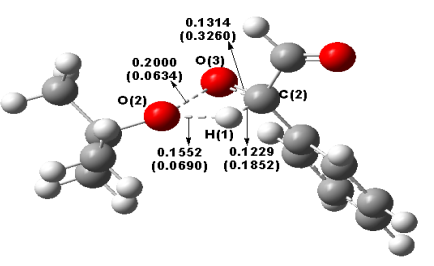

TS3

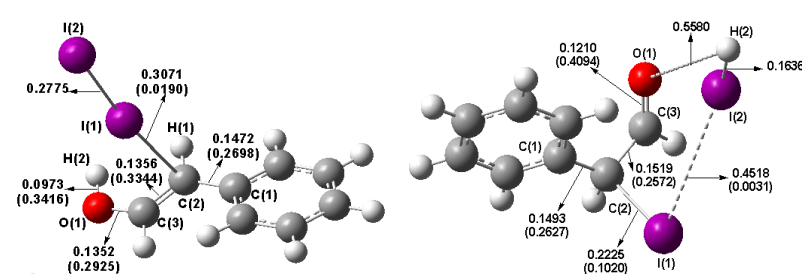

IM2

TS1

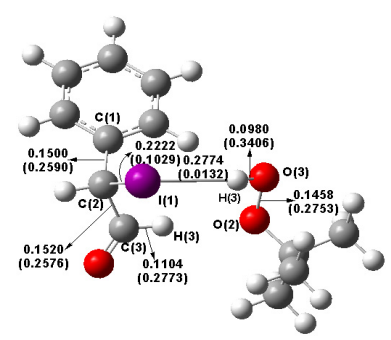

IM4

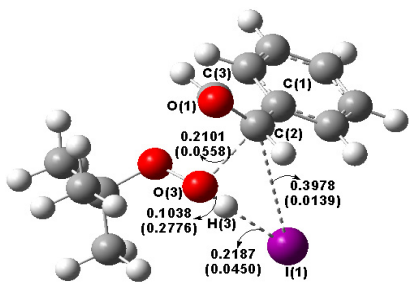

TS2

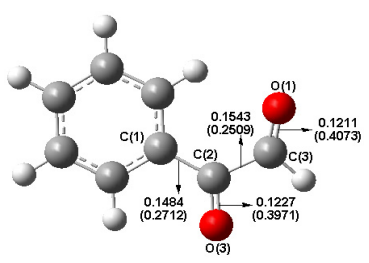

IM6

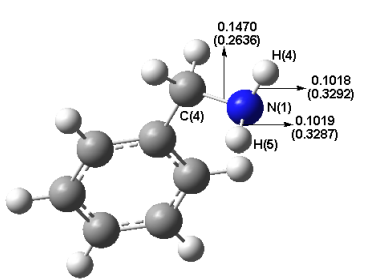

R2

(2)

IM7

TS4
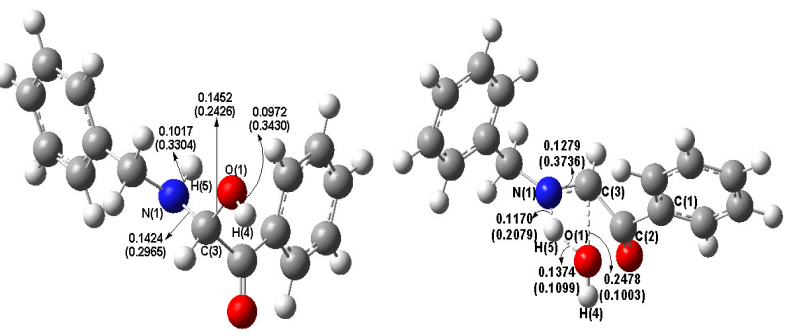

IM8

TS5

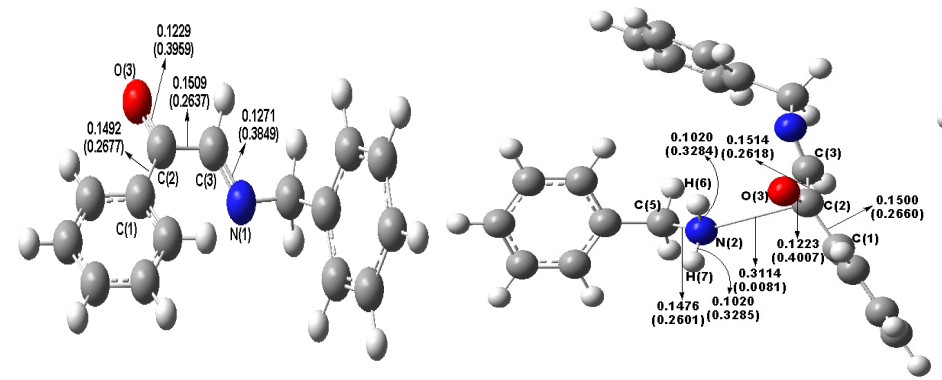

20.90\%

op

8

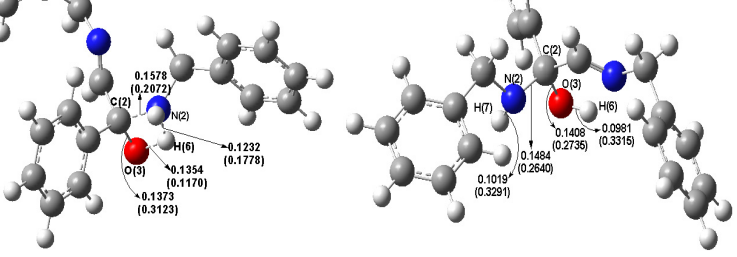

IM9

IM10

TS6

IM11

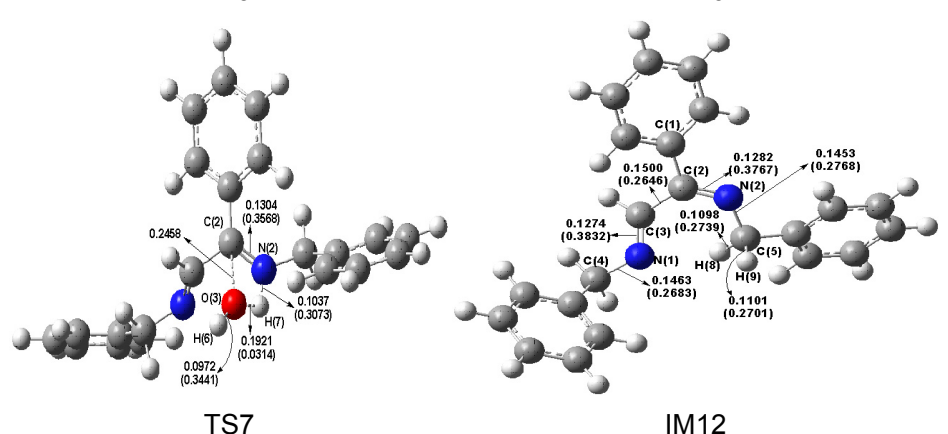

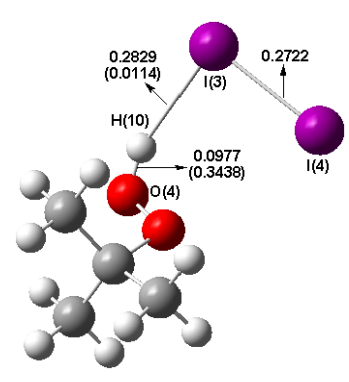

IM13

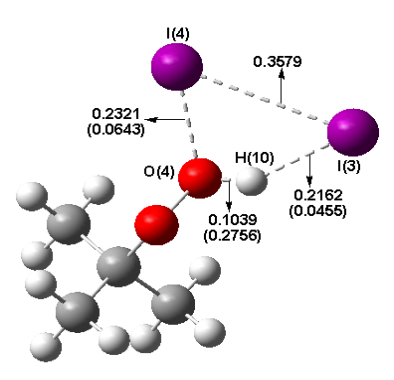

TS8

图 1 反应路径中各驻点的构型图(键长: $\mathrm{nm}$, 括号内为部分键鞍点电荷密度)

Figure 1 Geometric parameters of the compounds in the pathway (bond length in nm, BCP in a.u.) 


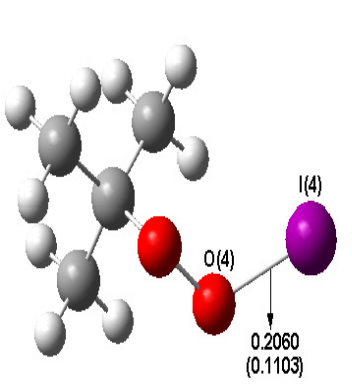

IM14

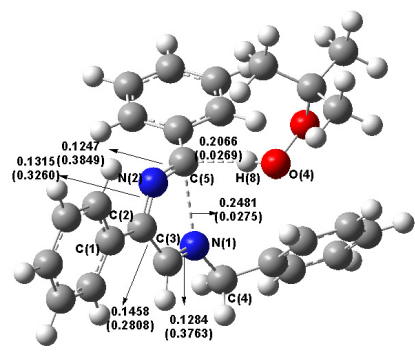

TS10

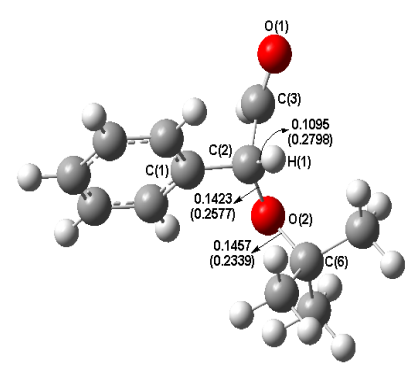

M2

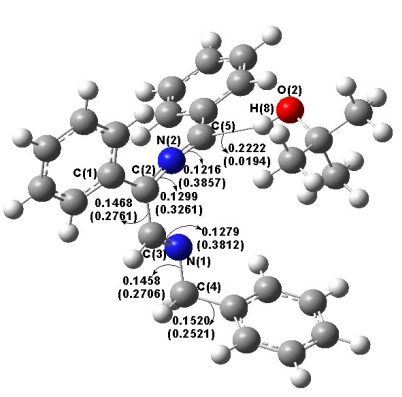

M4

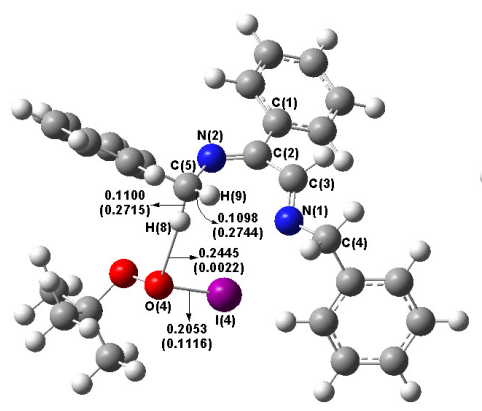

IM15

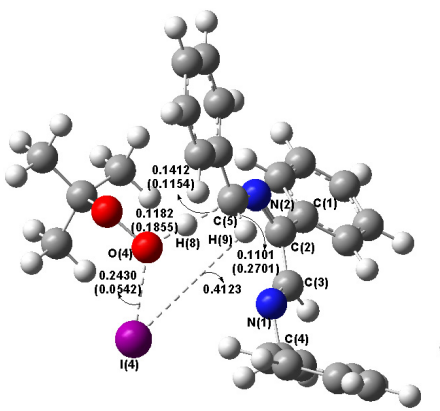

TS9

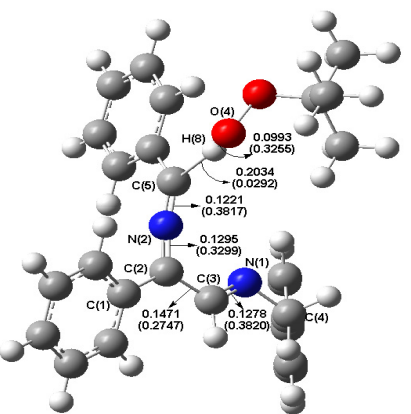

IM16
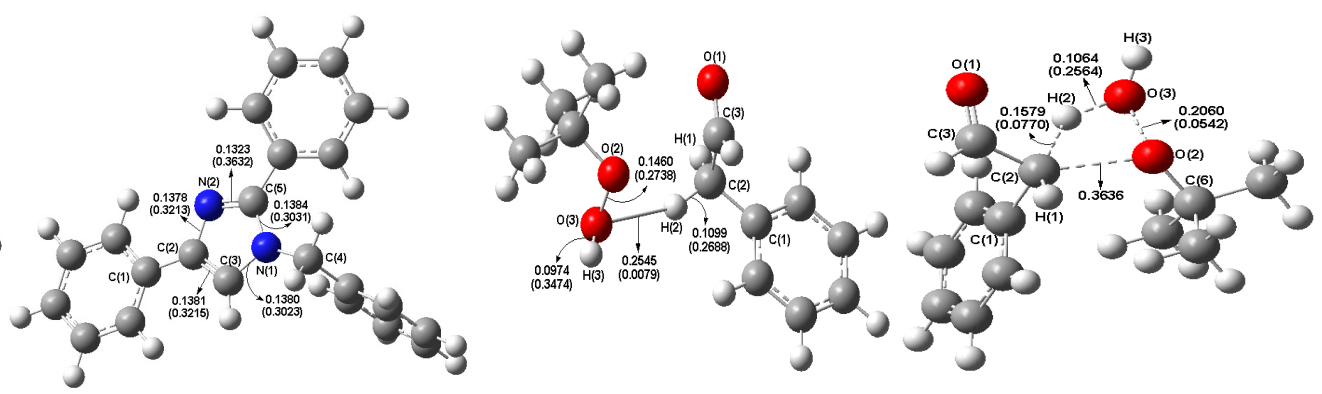

$P$

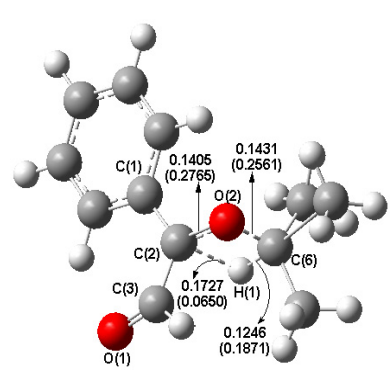

T2

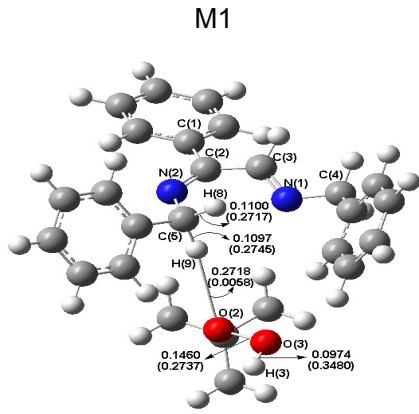

M3

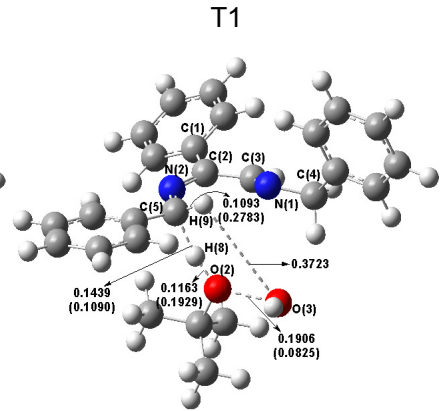

T3

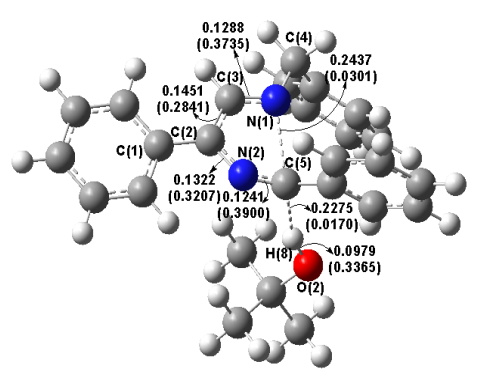

T4

图 2 反应路径中各驻点的分子构型图(键长: $\mathrm{nm}$, 括号内为部分键鞍点电荷密度)

Figure 2 Geometric parameters of the compounds in the pathway (bond length in nm, BCP in a.u.)

\section{$1.1 \mathrm{I}_{2}$ 催化反应机理}

碘催化苯乙醛和茮胺氧化环化合成多取代咪唑的 反应历程如 Scheme 1, 苯乙醛(R1)经共振变成烯醇式结 构 IM1, 烯醇式络合碘, 然后脱去 HI 分子形成中间体 IM3, 中间体 IM3 被氧化剂过氧叔丁醇 TBHP 氧化后形 成中间体 IM4, 中间体 IM4 脱去一个 $\mathrm{HI}$ 和 $\left(\mathrm{CH}_{3}\right)_{3} \mathrm{COH}$ 生成中间体 IM6, 中间体 IM6 与芐胺(R2)发生亲核加成 反应形成中间体 IM7, 中间体 IM7 通过互变异构再脱去
$\mathrm{H}_{2} \mathrm{O}$ 生成中间体 IM9, 中间体 IM9 再与芐胺(R2)发生亲 核加成反应形成中间体 IM10，中间体 IM10 同样通过互 变异构再脱去 $\mathrm{H}_{2} \mathrm{O}$ 生成中间体 IM12, 中间体 IM12 再次 被氧化剂过氧叔丁醇 TBHP 氧化作用生成中间体 IM15, 继而发生分子内环化, 先后脱去 $\mathrm{HI}, \mathrm{TBHP}$ 分子后形成 终产物 $(\mathrm{P})$.

卤素可与含活泼 $\alpha-\mathrm{H}$ 的醛或酮发生卤代反应，因 此，该反应的第一步反应物苯乙醛(R1)通过共振得到烯 
表 2 各驻点的活化能 $\Delta E\left(\mathrm{~kJ} \bullet \mathrm{mol}^{-1}\right)$ 和自由能 $\Delta G\left(\mathrm{~kJ} \bullet \mathrm{mol}^{-1}\right)$

Table 2 The activation energies $\Delta E\left(\mathrm{~kJ}^{\circ} \mathrm{mol}^{-1}\right)$ and the free energies $\Delta G\left(\mathrm{~kJ} \cdot \mathrm{mol}^{-1}\right)$ in the process

\begin{tabular}{|c|c|c|c|c|c|c|c|c|c|c|}
\hline \multirow{2}{*}{ Species } & \multicolumn{2}{|c|}{ Gas } & \multicolumn{2}{|c|}{$\mathrm{CH}_{3} \mathrm{CN}$} & \multicolumn{2}{|c|}{$\mathrm{C}_{2} \mathrm{H}_{5} \mathrm{OH}$} & \multicolumn{2}{|c|}{ DCE } & \multicolumn{2}{|c|}{ DMF } \\
\hline & $\Delta E$ & $\Delta G$ & $\Delta E$ & $\Delta G$ & $\Delta E$ & $\Delta G$ & $\Delta E$ & $\Delta G$ & $\Delta E$ & $\Delta G$ \\
\hline $\mathrm{IM} 2 \rightarrow \mathrm{TS} 1$ & 4.41 & 2.61 & 5.10 & 7.22 & 3.70 & 2.12 & 4.01 & 1.57 & 8.66 & 8.66 \\
\hline $\mathrm{IM} 4 \rightarrow \mathrm{TS} 2$ & 147.49 & 156.17 & 127.28 & 136.68 & 128.66 & 131.07 & 130.82 & 136.09 & 127.19 & 127.19 \\
\hline $\mathrm{IM} 5 \rightarrow \mathrm{TS} 3$ & 155.95 & 154.50 & 154.71 & 153.52 & 154.92 & 153.76 & 155.37 & 155.10 & 158.39 & 158.06 \\
\hline $\mathrm{IM} 7 \rightarrow \mathrm{TS} 4$ & 101.94 & 111.43 & 89.73 & 95.69 & 90.05 & 96.14 & 88.90 & 98.60 & 89.73 & 89.73 \\
\hline $\mathrm{IM} 8 \rightarrow \mathrm{TS} 5$ & 125.81 & 126.83 & 102.85 & 106.46 & 103.39 & 107.09 & 105.71 & 107.89 & 102.80 & 102.80 \\
\hline $\mathrm{IM} 10 \rightarrow \mathrm{TS} 6$ & 147.40 & 151.27 & 123.71 & 144.68 & 123.46 & 149.34 & 121.88 & 138.76 & 123.94 & 144.13 \\
\hline $\mathrm{IM} 11 \rightarrow \mathrm{TS} 7$ & 149.31 & 153.21 & 125.01 & 131.37 & 119.69 & 137.25 & 124.02 & 122.43 & 124.68 & 124.68 \\
\hline $\mathrm{IM} 13 \rightarrow \mathrm{TS} 8$ & 138.14 & 148.50 & 142.04 & 152.63 & 141.44 & 153.38 & 139.27 & 147.94 & 142.54 & 142.54 \\
\hline $\mathrm{IM} 15 \rightarrow \mathrm{TS} 9$ & 85.39 & 102.60 & 89.24 & 37.47 & 89.27 & 114.05 & 88.05 & 114.55 & 88.93 & 102.30 \\
\hline $\mathrm{IM} 16 \rightarrow \mathrm{TS} 10$ & 11.90 & 21.27 & 14.74 & 22.18 & 14.68 & 19.44 & 14.88 & 20.68 & 14.55 & 14.55 \\
\hline
\end{tabular}
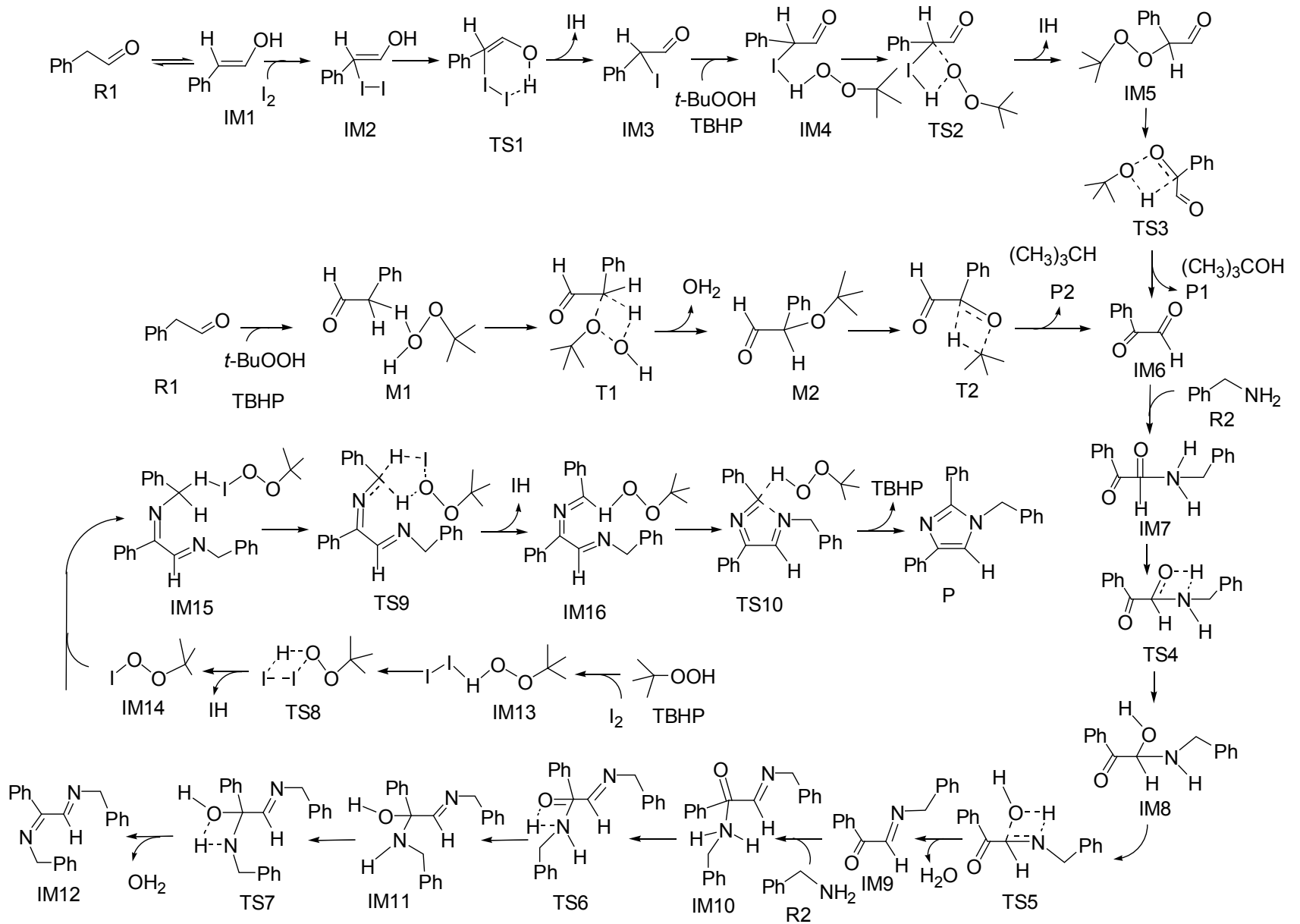

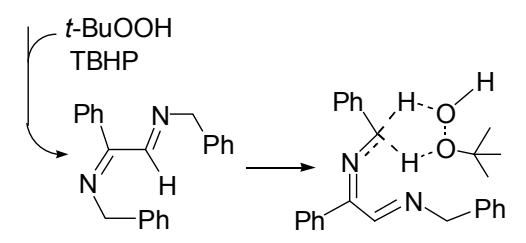

M3
T3

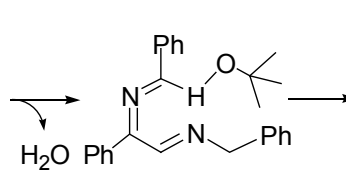

M4<smiles>CC(C)(C)OC(c1ccccc1)C(Cn1cnc(-c2ccccc2)c1)(c1ccccc1)C(C)(C)O</smiles>

$\mathrm{P} 1$

\section{Scheme 1}

醇互变异构体 IM1. 中间体 IM1 中 $\mathrm{C}(1)-\mathrm{C}(2), \mathrm{C}(2)$ $\mathrm{C}(3), \mathrm{C}(3)-\mathrm{O}(1)$ 的键长分别是 $0.1468,0.0134,0.1372$ $\mathrm{nm}$, 相应的 BCP 电荷密度分别为 $0.2717,0.3435,0.2789$ a.u. 催化剂 $\mathrm{I}_{2}$ 进攻中间体 IM1 中的 $\mathrm{C}(2)$ 形成中间体 IM2 
后, $\mathrm{I}(1)-\mathrm{I}(2), \mathrm{C}(1)-\mathrm{C}(2), \mathrm{C}(2)-\mathrm{C}(3)$ 键长分别增加 $0.0055,0.0004,0.1212 \mathrm{~nm}$, 相应的 BCP 电荷密度分别降 低了 $0.0032,0.0019,0.0091$ a.u., 说明 IM1 $\rightarrow \mathrm{IM} 2$ 过程 $\mathrm{C}(2)-\mathrm{C}(3)$ 键减弱双键被活化. NBO 计算结果得知, $\mathrm{BD}(2) \mathrm{C}(2)-\mathrm{C}(3) \rightarrow \mathrm{BD}^{*}(1) \mathrm{I}(1)-\mathrm{I}(2)$ 间的二阶稳定化能 $E(2)$ 为 $40.65 \mathrm{~kJ}^{\circ} \mathrm{mol}^{-1}, \mathrm{LP}(3) \mathrm{I}(1) \rightarrow \mathrm{BD}^{*}(2) \mathrm{C}(2)-\mathrm{C}(3)$ 间 的二阶稳定化能 $E(2)$ 为 $9.00 \mathrm{~kJ} \cdot \mathrm{mol}^{-1}$, 表明它们之间存 在较强的轨道间相互作用. IM $2 \rightarrow \mathrm{TS} 1 \rightarrow \mathrm{IM} 3$ 过程中, $\mathrm{O}(1)-\mathrm{H}(2)-\mathrm{I}(2)-\mathrm{I}(1)-\mathrm{C}(2)-\mathrm{C}(3)$ 形成六元环状过渡 态, 其环成键临界点(RCP)电荷密度为 0.015 a.u., $\mathrm{O}(1)$ $\mathrm{H}(2), \mathrm{I}(2)-\mathrm{I}(1)$ 键部分断裂, $\mathrm{H}(2)-\mathrm{I}(2)$ 键部分形成, 经 过渡态 TS1 后, HI 脱离形成中间体 IM3.

中间体 $\mathrm{IM} 3$ 中 $\mathrm{I}(1)-\mathrm{C}(2)$ 键长为 $0.2205 \mathrm{~nm}$, 过氧叔 丁醇 $\mathrm{TBHP}$ 中 $\mathrm{O}(3)-\mathrm{H}(3)$ 键长为 $0.0974 \mathrm{~nm}$, 中间体 IM3 与氧化剂 TBHP 结合形成的中间体 IM4 中 I(1)- $\mathrm{C}(2)$, $\mathrm{O}(3)-\mathrm{H}(3)$ 键长分别增加了 $0.0017,0.006 \mathrm{~nm}$, 相应的 BCP 电荷密度均有所降低. 同时 $\mathrm{I}(1)-\mathrm{H}(3)$ 的键长为 $0.2774 \mathrm{~nm}, \mathrm{BCP}$ 电荷密度为 0.0132 a.u., 由此可以看出, $\mathrm{I}(1)-\mathrm{H}(3)$ 键形成. 由 NBO 计算结果分析得知, LP(3) $\mathrm{I}(1) \rightarrow \mathrm{BD}^{*}(1) \mathrm{O}(3)-\mathrm{H}(3)$ 间的二阶稳定化能 $E(2)$ 为 $28.80 \mathrm{~kJ}^{2} \mathrm{~mol}^{-1}, \mathrm{BD}(2) \mathrm{C}(3)-\mathrm{O}(1) \rightarrow \mathrm{BD}^{*}(1) \mathrm{C}(2)-\mathrm{I}(1)$ 间 的二阶稳定化能 $E(2)$ 为 $12.73 \mathrm{~kJ} \cdot \mathrm{mol}^{-1}$, 表明它们之间 存在轨道间相互作用.中间体 IM4 经过渡态 TS2 脱去 HI 分子后形成中间体 IM5. 过渡态 TS2 中, C(2)-O(3)和 $\mathrm{I}(1)-\mathrm{H}(3)$ 键长分别是 0.2101 和 $0.2187 \mathrm{~nm}$, 比中间体 IM4 中键长分别减小 0.2243 和 $0.0587 \mathrm{~nm}, \mathrm{BCP}$ 电荷密 度增加 0.0558 和 0.0318 a.u., $\mathrm{I}(1)-\mathrm{C}(2)-\mathrm{O}(3)-\mathrm{H}(3)$ 之 间的 RCP 电荷密度 0.0067 a.u. 表明过渡态 TS2 为四元 环状过渡态, $\mathrm{C}(2)-\mathrm{O}(3)$ 部分成键, 且四元环过渡态使 $\mathrm{C}(2)-\mathrm{I}(1), \mathrm{O}(3)-\mathrm{H}(3)$ 键减弱. 中间体 IM5 中, C(2)$\mathrm{O}(3)$ 键长为 $0.1425 \mathrm{~nm}, \mathrm{BCP}$ 电荷密度为 0.2570 a.u., 表 明 $\mathrm{C}(2)-\mathrm{O}(3)$ 已完全成键, 后经过渡态 TS3 脱去叔丁醇 P1 分子形成中间体 IM6. 四元环过渡态 TS3 中, H(1) $\mathrm{C}(2)-\mathrm{O}(3)-\mathrm{O}(2)$ 之间的 $\mathrm{RCP}$ 电荷密度为 0.0422 a.u., $\mathrm{O}(3)-\mathrm{O}(2), \mathrm{H}(1)-\mathrm{C}(2)$ 键长分别是 $0.2000,0.1229 \mathrm{~nm}$, BCP 电荷密度分别是 $0.0634,0.1852$ a.u., 相比中间体 IM5 键长有所增加, BCP 电荷密度有所降低, $\mathrm{C}(2)-\mathrm{O}(3), \mathrm{H}(1)-\mathrm{O}(2)$ 键逐渐形成. 从表 1 数据分析可 知, IM5 $\rightarrow \mathrm{TS} 3 \rightarrow \mathrm{IM} 6$ 过程所需活化能为 $155.95 \mathrm{~kJ} \cdot \mathrm{mol}^{-1}$, 是该反应的速控步骤。中间体 IM6 中, $\mathrm{H}(1)-\mathrm{C}(2)$, $\mathrm{O}(3)-\mathrm{O}(2)$ 键已完全断裂, $\mathrm{C}(2)-\mathrm{O}(3)$ 键长为 $0.1227 \mathrm{~nm}$, $\mathrm{BCP}$ 电荷密度为 0.3971 a.u., 说明脱去叔丁醇 $\mathrm{P} 1$ 分子后 $\mathrm{C}(2)-\mathrm{O}(3)$ 键进一步增强.

反应物芐胺 $(\mathrm{R} 2)$ 中 $\mathrm{N}$ 原子进攻中间体 IM6 中 $\mathrm{C}(3)$ 原子，导致 $\mathrm{N}(1)-\mathrm{H}(4), \mathrm{N}(1)-\mathrm{H}(5), \mathrm{C}(3)-\mathrm{O}(1), \mathrm{C}(4)-$
$\mathrm{N}(1)$ 键长均有所增加, 形成中间体 IM7 后 $\mathrm{N}(1)$ - $\mathrm{C}(3)$ 键 长为 $0.2861 \mathrm{~nm}, \mathrm{BCP}$ 电荷密度为 0.0141 a.u. 从表 1 数 据看出, IM6 $\rightarrow \mathrm{IM} 7$ 过程能量降低了 $10.87 \mathrm{~kJ} \cdot \mathrm{mol}^{-1}$, 中 间体 IM7 能稳定存在. IM7 $\rightarrow \mathrm{TS} 4 \rightarrow \mathrm{IM} 8$ 过程中, 过渡态 TS4 较中间体 IM7 中 $\mathrm{N}(1)-\mathrm{H}(4), \mathrm{O}(1)-\mathrm{H}(4)$ 键长分别 增加 $0.0220,0.2582 \mathrm{~nm}$, 相应的 $\mathrm{BCP}$ 电荷密度降低 0.1549, 0.1131 a.u., 表明过渡态 TS4 中 $\mathrm{N}(1)-\mathrm{H}(4)$ 键部 分断裂, 且 $\mathrm{O}(1)-\mathrm{H}(4)$ 键部分形成. 中间体 IM8 中, $\mathrm{N}(1)-\mathrm{H}(5), \mathrm{C}(3)-\mathrm{O}(1), \mathrm{N}(1)-\mathrm{C}(3)$ 键长分别是 0.1017 , $0.1452,0.1424 \mathrm{~nm}, \mathrm{BCP}$ 电荷密度分别是 $0.3304,0.2426$, 0.2965 a.u., 与之相比, 过渡态 TS5 中 $\mathrm{N}(1)-\mathrm{H}(5)$, $\mathrm{C}(3)-\mathrm{O}(1), \mathrm{N}(1)-\mathrm{C}(3)$ 键长分别增加 $0.0153,0.1026$, $0.0205 \mathrm{~nm}, \mathrm{BCP}$ 电荷密度分别降低 $0.1225,0.1223$, 0.0771 a.u., 中间体 IM9 中 $\mathrm{N}(1)-\mathrm{C}(3)$ 键长为 $0.1271 \mathrm{~nm}$, BCP 电荷密度 0.3849 a.u., 中间体 IM9 的(1)- $C$ (3) 双键 形成. 从表 1 数据可知, IM8 $8 \rightarrow \mathrm{TS} 5 \rightarrow \mathrm{IM} 9$ 过程所需活化 能为 $125.81 \mathrm{~kJ} \cdot \mathrm{mol}^{-1}$, 该反应比较容易发生. 紧接着另 一分子反应物(R2)中 $\mathrm{N}$ 原子继续进攻中间体 IM9 中 $\mathrm{C}(2)$ 原子形成中间体 IM10, 其中 $\mathrm{C}(2)-\mathrm{N}(2)$ 键长为 0.3114 $\mathrm{nm}, \mathrm{BCP}$ 电荷密度为 0.0081 a.u.. 中间体 IM10 经过渡态 TS6 形成中间体 IM11, 过渡态 TS6 中 $\mathrm{N}(2)-\mathrm{H}(6)$, $\mathrm{O}(3)-\mathrm{H}(6)$ 键长为 $0.1232,0.1354 \mathrm{~nm}, \mathrm{BCP}$ 电荷密度为 $0.1778,0.1170$ a.u., 相比中间体 IM10 键长分别增加了 $0.0212,0.1424 \mathrm{~nm}, \mathrm{BCP}$ 电荷密度分别增加了 0.1170 , 0.1506 a.u. 表明过渡态 TS6 中 $\mathrm{O}(3)-\mathrm{H}(6)$ 键部分断裂, $\mathrm{N}(2)-\mathrm{H}(6)$ 键部分形成. 中间体 IM11 中 $\mathrm{O}(3)-\mathrm{H}(6)$ 键 完全断裂, 后经过渡态 TS7 脱去 $\mathrm{H}_{2} \mathrm{O}$ 分子形成中间体 IM12。过渡态 TS7 中, $\mathrm{C}(2)-\mathrm{O}(3)$ 和 $\mathrm{N}(2)-\mathrm{H}(7)$ 键长为 0.2458 和 $0.1037 \mathrm{~nm}$ ，相比中间体 IM11 中增加了 0.1050 和 $0.0018 \mathrm{~nm} ; \mathrm{N}(2)-\mathrm{C}(2)$ 键长为 $0.1304 \mathrm{~nm}, \mathrm{BCP}$ 电荷密 度为 0.3568 a.u., 表明过渡态 TS7 中 $\mathrm{C}(2)-\mathrm{O}(3)$ 键减弱, $\mathrm{N}(2)-\mathrm{C}(2)$ 键增强. 中间体 IM12 中 $\mathrm{N}(2)-\mathrm{C}(2)$ 键长为 $0.1282 \mathrm{~nm}, \mathrm{BCP}$ 电荷密度为 0.3767 a.u., 表明 $\mathrm{N}(2)-$ $\mathrm{C}(2)$ 双键已完全形成. 过氧叔丁醇 TBHP 与催化剂 $\mathrm{I}_{2}$ 形 成中间体 IM13, 由于 $\mathrm{I}(3)$ 与 $\mathrm{H}(10)$ 相互靠近导致 $\mathrm{O}(4)$ $\mathrm{H}(10), \mathrm{I}(3)-\mathrm{I}(4)$ 键长有所增加. 中间体 IM13 经过渡态 TS8 脱去 HI 分子形成中间体 IM14. 中间体 IM14 中 O(4) 原子与中间体 IM12 中 $\mathrm{H}(8)$ 原子相互作用形成中间体 IM15. 表 1 的能量数据分析可知, 该过程能量降低了 $155.96 \mathrm{~kJ} \cdot \mathrm{mol}^{-1}$, 中间体 IM15 稳定存在. 中间体 IM15 经五元环过渡态 TS9 脱去 HI 分子形成中间体 IM16. 与 中间体 IM14 相比, 过渡态 TS9 中 $\mathrm{O}(4)-\mathrm{I}(4), \mathrm{C}(5)$ $\mathrm{H}(9)$ 键长分别增加了 $0.0377,0.0002 \mathrm{~nm}, \mathrm{BCP}$ 电荷密度 分别降低了 $0.0576,0.0043$ a.u., 该过程的活化能为 $85.39 \mathrm{~kJ} \cdot \mathrm{mol}^{-1}$. 中间体 IM16 经过渡态 TS10 发生分子内 
环化脱去 TBHP 分子形成产物(P). 研究发现, IM13 $\rightarrow$ $\mathrm{TS} 8 \rightarrow \mathrm{IM} 14$ 逆反应过程的活化能相对较低, 且为 49.58 $\mathrm{kJ} \cdot \mathrm{mol}^{-1}$, 反应过程中生成的 $\mathrm{HI}$ 在过氧叔丁醇存在时, 可以通过 $\mathrm{IM} 14 \rightarrow \mathrm{TS} 8 \rightarrow \mathrm{IM} 13$ 过程还原为磑单质.

\section{2 无 $\mathrm{I}_{2}$ 催化反应机理}

我们同时研究了无催化剂 $\mathrm{I}_{2}$ 参与下的反应历程, 反 应历程见 Scheme 1, 反应物苯乙醛与过氧叔丁醇作用形 成中间体 M1. 中间体 $\mathrm{M} 1$ 经过四元环过渡态 $\mathrm{T} 1$ 脱去 $\mathrm{H}_{2} \mathrm{O}$ 分子形成中间体 $\mathrm{M} 2$. 中间体 $\mathrm{M} 2$ 经过渡态 $\mathrm{T} 2$ 脱去 叔丁烷分子 P2 形成中间体 IM6, IM6 $\rightarrow$ IM12 这一过程与 有碘参与时完全相同. 由表 1 中的能量数据分析可知, $\mathrm{M} 2 \rightarrow \mathrm{T} 2 \rightarrow \mathrm{IM} 6$ 步骤在气相条件下的活化能是 514.32 $\mathrm{kJ} \cdot \mathrm{mol}^{-1}$, 为该反应通道的速控步骤; 而有碘参与时, 催化剂碘使苯乙醛乙基上的 $\mathrm{C}-\mathrm{H}$ 键活化, 使得中间体 IM5 经过渡态 TS3 得到中间体 IM6 过程中所需要的活 化能为 $145.94 \mathrm{~kJ} \cdot \mathrm{mol}^{-1}$, 活化能显著降低. 中间体 IM12 与 TBHP 结合形成中间体 $\mathrm{M} 3$, 再经过渡态 $\mathrm{T} 3$ 脱去 $\mathrm{H}_{2} \mathrm{O}$ 分子形成中间体 $\mathrm{M} 4$, 由表 1 数据分析可知 $\mathrm{M} 3 \rightarrow \mathrm{T} 3 \rightarrow$ M4 过程的活化能为 $164.41 \mathrm{~kJ} \cdot \mathrm{mol}^{-1}$. 中间体 M4 经过渡 态 $\mathrm{T} 4$ 脱去叔丁醇分子 $\mathrm{P} 1$ 得到产物 $\mathrm{P}$, 该过程的活化能

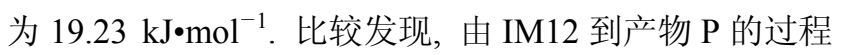
中, 有碘参与时, IM13 $\rightarrow \mathrm{TS} 8 \rightarrow \mathrm{IM} 14$ 步骤的活化能最高 且为 $144.17 \mathrm{~kJ} \cdot \mathrm{mol}^{-1}$; 而无碘参与时, 活化能最高的步 骤为 $\mathrm{M} 3 \rightarrow \mathrm{T} 3 \rightarrow \mathrm{M} 4$. 由此, 进一步说明 $\mathrm{I}_{2}$ 有效地促进了 反应的进行.

\section{3 能量分析}

由表 1 中能量数据可以看出, 反应物(R1)与中间体 IM1 是烯醇互变过程, 中间体 IM1 与催化剂 $\mathrm{I}_{2}$ 结合形成 中间体 IM2 过程, 能量升高了 $1.97 \mathrm{~kJ} \cdot \mathrm{mol}^{-1}$, 表明中间 体 IM2 能稳定存在. 在催化剂 $\mathrm{I}_{2}$ 参与复杂反应过程中, 通过我们上面对机理的分析发现: IM5 $\rightarrow \mathrm{TS} 3 \rightarrow \mathrm{IM} 6$ 过程 的活化能为 $145.94 \mathrm{~kJ} \cdot \mathrm{mol}^{-1}$, 表明过氧叔丁醇的氧化过 程需要较高的活化能, 为该反应的速控步骤. 从表 1 可 以看出, 气相条件下无催化剂 $\mathrm{I}_{2}$ 参与反应, $\mathrm{M} 2 \rightarrow$ $\mathrm{T} 2 \rightarrow \mathrm{M} 3$ 为速控步骤, 活化能为 $514.32 \mathrm{~kJ} \cdot \mathrm{mol}^{-1}$, 在无 碘催化情况下, 表明反应有很高的活化能, 反应不能发 生. 比较可知, 催化剂 $\mathrm{I}_{2}$ 参与反应可使活化能显著降低, 说明催化剂 $\mathrm{I}_{2}$ 有效地促进了反应的进行. 在有碘催化和 无碘催化两种情况下, IM6 $+\mathrm{R} 2 \rightarrow \mathrm{IM} 7 \rightarrow \mathrm{TS} 4 \rightarrow \mathrm{IM} 8 \rightarrow$ $\mathrm{TS} 5 \rightarrow \mathrm{IM} 9 \rightarrow \mathrm{IM} 10 \rightarrow \mathrm{TS} 6 \rightarrow \mathrm{IM} 11 \rightarrow \mathrm{TS} 7 \rightarrow \mathrm{IM} 12$ 过程完全 一致, 两种情况下反应的速控步骤均不在此过程中.

\section{4 溶剂的影响}

汪志勇小组 ${ }^{[13]}$ 在进行反应条件优化时, 分别选取 了 $\mathrm{CH}_{3} \mathrm{CN}, \mathrm{C}_{2} \mathrm{H}_{5} \mathrm{OH}, \mathrm{DCE}$ 和 DMF 四种溶剂进行实验,
产率分别为 $73 \%, 69 \%, 61 \%, 58 \%$. 在不同溶剂中该反应 所得产率不同，为了探讨溶剂化作用的效果，我们采用 连续介质模型, 在以上 4 种溶剂条件下对反应过程中各 驻点进行了全参数优化和能量分析. 表 2 中我们列出了 反应活化步骤的能量和自由能变化. 从表 2 看出: 在溶 剂化条件下大部分过程的活化能均有所降低, 气相条件 与溶剂化条件下的速控步骤一致, 均为中间体 IM5 $\rightarrow$ 过 渡态 TS3 过程. 不同溶剂化下速控步骤的活化能比较接 近, 这与实验所得产率相近是一致的, 不同溶剂化下速 控步骤的自由能变化大小与产率大小顺序相符, 我们的 计算结果与实验吻合.

我们发现汪志勇小组笁选实验条件时, 溶剂极性对 产率影响较大, 以上 4 种溶剂的极性大小为: DMF (6.40) $>\mathrm{CH}_{3} \mathrm{CN}(6.20)>\mathrm{C}_{2} \mathrm{H}_{5} \mathrm{OH}(4.30)>\mathrm{DCE}$ (3.40), 他 们的实验结果显示, 反应时所选溶剂极性越大, 产率越 高, 只有使用 DMF 溶剂时出现了偏差. 为了从理论上 考察溶剂对该反应的影响, 我们选取了极性为 7.20 的有 机溶剂二甲亚砜(DMSO)对该反应的速控步骤(中间体 IM5 $\rightarrow$ 过渡态 TS3)进行计算, 计算结果表明, IM5 $\rightarrow$ TS3 过程中 $\Delta E=154.61 \mathrm{~kJ} \cdot \mathrm{mol}^{-1}, \Delta G=153.24 \mathrm{~kJ} \cdot \mathrm{mol}^{-1}$, 均 比 DMF, $\mathrm{CH}_{3} \mathrm{CN}, \mathrm{C}_{2} \mathrm{H}_{5} \mathrm{OH}, \mathrm{DCE}$ 溶剂下的活化能和自由 能变化值小, 由此我们可以从理论上预测, 该反应若使 用 DMSO 溶剂可能获得更高的产率.

\section{2 结论}

本文采用密度泛函理论在 B3LYP/6-31+G*基组水 平上研究了碘催化氧化环化合成多取代咪唑的微观反 应机理, 研究发现可能的反应通道为 $\mathrm{R} 1 \rightarrow \mathrm{IM} 1+$ $\mathrm{I}_{2} \rightarrow \mathrm{IM} 2 \rightarrow \mathrm{TS} 1 \rightarrow \mathrm{IM} 3+\mathrm{TBHP} \rightarrow \mathrm{IM} 4 \rightarrow \mathrm{TS} 2 \rightarrow \mathrm{IM} 5 \rightarrow \mathrm{TS} 3 \rightarrow$ $\mathrm{IM} 6+\mathrm{R} 2 \rightarrow \mathrm{IM} 7 \rightarrow \mathrm{TS} 4 \rightarrow \mathrm{IM} 8 \rightarrow \mathrm{TS} 5 \rightarrow \mathrm{IM} 9 \rightarrow \mathrm{IM} 10 \rightarrow \mathrm{TS} 6 \rightarrow$ $\mathrm{IM} 11 \rightarrow \mathrm{TS} 7 \rightarrow \mathrm{IM} 12+\mathrm{IM} 14\left(\mathrm{TBHP}+\mathrm{I}_{2} \rightarrow \mathrm{TS} 8 \rightarrow \mathrm{IM} 14\right) \rightarrow$ $\mathrm{IM} 15 \rightarrow \mathrm{TS} 9 \rightarrow \mathrm{IM} 16 \rightarrow \mathrm{TS} 10 \rightarrow \mathrm{P}$, 对反应各驻点的能量分 析结果表明, IM5 $\rightarrow \mathrm{TS} 3 \rightarrow \mathrm{IM} 6$ 是该反应的速控步骤, 活 化能为 $155.95 \mathrm{~kJ} \cdot \mathrm{mol}^{-1}$. 无碘催化剂参与反应时, $\mathrm{M} 2 \rightarrow \mathrm{T} 2 \rightarrow \mathrm{M} 3$ 为速控步骤, 活化能为 $518.72 \mathrm{~kJ} \cdot \mathrm{mol}^{-1}$, 说明在无催化剂 $\mathrm{I}_{2}$ 参与反应时, 该反应几乎不能发生, 从而进一步证实了催化剂 $\mathrm{I}_{2}$ 有效地促进了该反应的进 行. 有碘催化和无碘催化两种情况下, IM6 $\rightarrow$ IM12 过程 完全一致. 我们计算了 4 种溶剂对反应过程的影响, 计 算结果表明气相与溶剂化条件下的速控步骤一致, 均为 IM5 $\rightarrow$ TS3 过程, 溶剂化下活化能有降低的趋势, 溶剂 化下速控步骤活化能大小顺序为: $\mathrm{CH}_{3} \mathrm{CN}<\mathrm{C}_{2} \mathrm{H}_{5} \mathrm{OH}<$ $\mathrm{DCE}<\mathrm{DMF}$ ，与实验给出的产率分别为 $73 \%, 69 \%, 61 \%$, $58 \%$ 相吻合. 根据溶剂极性变化特征，我们从理论上预 测，该反应若使用 DMSO 溶剂可能获得更高的产率. 


\section{3 计算方法}

采用密度泛函理论(DFT) 中的 B3LYP 方法, 在 $6-31+\mathrm{G}(\mathrm{d})$ 基组水平下 ${ }^{[20,21]}$ (I 采用 MIDIX 基组), 对碘 催化苯乙醛和苠胺发生氧化环化合成多取代咪唑体系 中所有反应物、中间体、过渡态和产物的结构进行优化. 通过频率计算确认过渡态有唯一虚频, 内毫反应坐标 (IRC)计算以及振动频率分析进一步验证了过渡态的合 理性, 并在相同基组水平上运用自然键价轨道(NBO) $)^{[22]}$ 方法分析了部分中间体的轨道间相互作用. 为了提高计 算精度, 在 6-311++ G(d,p) 基组水平上对优化后的各 化合物进行了单点能计算和零点能矫正. 利用 AIM2000 程序包 ${ }^{[23]}$ 计算了相应的成键临界点(BCP)和成 环临界点(RCP) 电荷密度, 分析成键特征, 通过振动分 析得到零点能(ZPE)及其校正后的总能量. 为了对比实 验结果, 我们在相同基组水平下采用自洽场(SCRF)连 续介质模型 $(\mathrm{PCM})^{[24]}$ 对反应过程的中间体、过渡态进行 了全参数优化, 分析了溶剂化作用. 以上计算采用 Gaussian 09 程序 ${ }^{[25]}$ 完成.

\section{References}

[1] Zhou, B. Y.; Zhang, J.; Li, X. N.; She, M. Y.; Zhang, J.; Li, J. L.; Shi, Z. Chin. J. Org. Chem. 2013, 33, 423 (in Chinese). (周葆悦, 张金, 李向南, 厍梦尧, 张劲, 李剑利, 史真, 有机化 学, 2013, 33, 423.)

[2] Palani, T.; Appaswami, L.; Pirama, N. A. Tetrahedron Lett. 2010, $51,2813$.

[3] De la Fuente, V.; Fleury-Bregeot, N.; Castillon, S.; Claver, C. Green Chem. 2012, 14, 2715

[4] Karami, B.; Eskandari, K.; Ghasemi, A. Turk. J. Chem. 2012, 36, 601.

[5] Zhang, J.; Wang, X.; Yang, M. P.; Wan, K. R.; Yin, B.; Wang, Y. X.; Li, J. L.; Shi, Z. Tetrahedron Lett. 2011, 52, 1578.

[6] Mazaahir, K.; Pooman, M. Tetrahedron Lett. 2006, 47, 5029.

[7] Huang, H. W.; Ji, X. C.; Wu, W. Q.; Jiang, H. F. Adv. Synth. Catal. 2013, 355, 170

[8] Zhou, Y.; Yan, P. F.; Li, G. M.; Chen, Z. J. Chin. J. Org. Chem. 2009, 29, 1719 (in Chinese)

(周颖, 间鹏飞, 李光明, 陈正军, 有机化学, 2009, 29, 1719.)

[9] Zhang, Z. H.; Liu, Q. B. Prog. Chem. 2006, 18, 270 (in Chinese). (张占辉, 刘庆涁, 化学进展, 2006, 18, 270.)

[10] Shen, S. S.; Xu, X. P.; Ji, S. J. Chin. J. Org. Chem. 2009, 29, 806 (in Chinese).

(沈舒苏, 徐小平, 纪顺俊, 有机化学, 2009, 29, 806.)

[11] Ren, Y. M.; Cai, C.; Yang, R. C. RSC Adv. 2013, 27, 7182

[12] Abu, T. K.; Arindam, G.; Md, M. K. Tetrahedron Lett. 2012, 53, 2622.

[13] Zhang, B. Q.; Wan, C. F.; Wang, Q.; Zhang, S.; Zha, Z. G.; Wang, Z. Y. Acta Chim. Sinica 2012, 70, 2408 (in Chinese).

(张百群, 万常峰, 王强, 张帅, 查正根, 汪志勇, 化学学报, 2012, 70, 2408.)

[14] Alcaide, B.; Almendros, P.; Cabrero G.; Callejo R.; Ruiz, P. M.; Arnó, M.; Domingo, R. L. Adv. Synth. Catal. 2010, 352, 1688.

[15] Lebøuf, D.; Gandon, V.; Ciesielski, J.; Frontier, J. A. J. Am. Chem. Soc. 2012, 134, 6296.

[16] Wang, G.; Cai, W. F.; Li, L. C.; Tian, A. M. Sci. China Chem. 2013, 43, 185 (in Chinese).

(王刚, 蔡睆飞, 李来才, 田安民，中国科学：化学， 2013，43, 185.)

[17] Wang, G.; Bai, K. K.; Li, L. C.; Tian, A. M. Sci. China Chem. 2013, 44, 334 (in Chinese).

(王刚, 白坤坤, 李来才, 田安民, 中国科学: 化学, 2013，44, 334.)

[18] McKeown, B. A.; Gonzalez, H. E.; Friedfeld, M. R.; Gunnoe, T. B.; Cundari, T. R.; Sabat, M. J. Am. Chem. Soc. 2011, 133, 19131.

[19] Yuan, Q. L.; Zhou, X. T.; Ji, H. B. Catal. Commun. 2010, 12, 202.

[20] Lee, C.; Yang, W.; Parr, R. G. Phys. Rev. B 1988, 37, 785.

[21] Miehlich, B.; Savin, A.; Stoll, H.; Preuss, H. Chem. Phys. Lett. 1989, 157, 200.

[22] Reed, A. E.; Weinhold, F.; Curtiss, L. A.; Pochatko, D. J. J. Chem. Phys. 1986, 84, 5687.

[23] Bader, R. W. F. Atoms in Molecules, A Quantum Theory, Oxford University Press, Oxford, 1990.

[24] Barone, V.; Cossi, M. J. Phys. Chem. A 1998, 102, 1995.

[25] Frisch, M. J.; Trucks, G. W.; Schlegel, H. B.; Scuseria, G. E.; Robb, M. A.; Cheeseman, J. R.; Scalmani, G.; Barone, V.; Mennucci, B.; Petersson, G. A.; Nakatsuji, H.; Caricato, M.; Li, X.; Hratchian, H. P.; Izmaylov, A. F.; Bloino, J.; Zheng, G.; Sonnenberg, J. L.; Hada, M.; Ehara, M.; Toyota, K.; Fukuda, R.; Hasegawa, J.; Ishida, M.; Nakajima, T.; Honda, Y.; Kitao, O.; Nakai, H.; Vreven, T.; Montgomery, J. J. A; Peralta, J. E.; Ogliaro, F.; Bearpark, M.; Heyd, J. J.; Brothers, E.; Kudin, K. N.; Staroverov, V. N.; Kobayashi, R.; Normand. J.; Raghavachari, K.; Rendell, A.; Burant, J. C.; Iyengar, S. S.; Tomasi, J.; Cossi, M.; Rega, N.; Millam, J. M.; Klene, M.; Knox, J. E.; Cross, J. B.; Bakken, V.; Adamo, C.; Jaramillo, J.; Gomperts, R.; Stratmann, R. E.; Yazyev, O.; Austin, A. J.; Cammi, R.; Pomelli, C.; Ochterski, J. W.; Martin, R. L.; Morokuma, K.; Zakrzewski, V. G.; Voth, G. A.; Salvador, P.; Dannenberg, J. J.; Dapprich, S.; Daniels, A. D.; Farkas, Ö.; Foresman, J. B.; Ortiz, J. V.; Cioslowski, J.; Fox, D. J. Gaussian 09, Revision A.02, Gaussian, Inc., Wallingford CT, 2009. 